\title{
Artigo/Article
}

\section{Associação entre incidência de dengue, pluviosidade e densidade larvária de Aedes aegypti, no Estado de Goiás}

\author{
Association between dengue incidence, rainfall and larval density of Aedes aegypti, in the State \\ of Goiás
}

\author{
Sócrates Siqueira de Souza ${ }^{1,2}$, Ionizete Garcia da Silva ${ }^{1,3}$ e Heloísa Helena Garcia da Silva ${ }^{1,3}$
}

\begin{abstract}
RESUMO
Introdução: A densidade larvária de Aedes aegypti flutua de acordo com as variações climáticas sazonais, elevando-se nas estações de maior pluviosidade, em função do número de potenciais criadouros disponíveis, o que predispõe ao aumento da incidência de dengue. Este estudo teve o objetivo de mostrar a associação entre os casos de dengue, a pluviosidade e o índice de infestação predial. Métodos: Os municípios foram estratificados de acordo com transmissão e risco de dengue, e infestados ou não pelo mosquito. Utilizou-se o índice de infestação predial larvário (IIP) como indicador de risco de transmissão. Resultados: Houve correlação positiva entre o IIP, o número de casos de dengue e a pluviosidade. A transmissão da doença foi maior nos quatro primeiros meses de cada ano estudado, período de elevada pluviosidade, diminuindo, nos meses de junho a setembro, época de poucas chuvas. Os casos de dengue mostraram-se contínuos e crescentes nos meses de janeiro a março de cada ano, declinando nos meses de abril e maio, quando ocorreu a interrupção na maioria dos municípios. A região metropolitana de Goiânia foi responsável por mais de $80 \%$ dos casos de dengue em Goiás e a transmissão foi contínua em todos os meses, embora baixa no período de maio a dezembro, mas com aumento nos três últimos meses, os quais, normalmente, apresentam índices baixos de transmissão. Conclusões: A correlação positiva entre o IIP e a pluviosidade, e o IIP e a incidência de casos, apontaram para uma associação significativa crescente na transmissão e no número de casos de dengue.
\end{abstract}

Palavras-Chaves: Aedes aegypti. Dengue. Pluviosidade. Associação.

\begin{abstract}
Introduction: The larval density of Aedes aegypti fluctuates according to seasonal climatic changes. It rises in seasons with higher rainfall, according to the number of potential breeding sites available, thereby predisposing towards increased incidence of dengue. This study aimed to show the association between dengue cases, rainfall and the building infestation rate. Methods: The municipalities were stratified according to the risk and transmission of dengue, and according to presence or absence of mosquito infestation. We used the building larval infestation rate as an indicator for the risk of transmission. Results: There was a positive correlation between the building infestation rate, number of dengue cases and rainfall. Disease transmission was greater during the first four months of each year studied (period of high rainfall) and lower between June and September (season with low rainfall). Dengue cases occurred continually and in increased numbers between January and March of each year, and then declined in the months of April and May, when it ceased in most municipalities. The metropolitan region of Goiânia was responsible for more than $80 \%$ of the dengue cases in Goiás. Transmission was continuous throughout the year, although lower during the period from May to December. Over the last three months, there was increased transmission, but usually still at low rates. Conclusions: The positive correlation between the building infestation rate and rainfall, and between this rate and the incidence of cases, indicated a significant association between increasing transmission and increasing numbers of dengue cases.
\end{abstract}

Key-words: Aedes aegypti. Dengue. Rainfall. Correlation.

1. Curso de Pós-Graduação em Medicina Tropical, Instituto de Patologia Tropical e Saúde Pública, Universidade Federal de Goiás. Goiânia GO. 2. Superintendência de Políticas de Atenção Integral à Saúde, Secretaria de Estado da Saúde de Goiás. 3. Laboratórios de Biologia e Fisiologia de Insetos e de Bioatividade de Plantas, Instituto de Patologia Tropical e Saúde Pública, Universidade Federal de Goiás, Goiânia, GO.

Endereço para correspondência: Dr. Ionizete Garcia da Silva. Dept ${ }^{\underline{o}}$ Microbiologia, Imunologia, Parasitologia e Patologia/IPTSP/UFG. Caixa Postal 131, 74001-970 Goiânia, GO.

Tel:55 62 209-6128; Fax: 5562 261-2077.

e-mail:ionizete@iptsp.ufg.br

Recebido para publicação em 13/03/2009

Aceito em 05/03/2010

\section{INTRODUÇÃO}

Aedes aegyptié considerado o vetor mais importante de dengue. Adaptado ao ambienteurbano, essemosquito vive em contato com cerca de 2,5 bilhões de pessoas ${ }^{1}, o$ que os expõem ao risco da transmissão de dengue.

Em Goiás, esse mosquito introduziu-se em 1987, no sul do estado. O comportamento sinantrópico e antropofílico desse vetor possibilitou sua expansão geográfica e, em 1990, foi descrito pela primeira vez em Goiânia ${ }^{2}$, cinco anos depois, 59 municípios registravam epidemias de dengue. A partir daí, a doença mostrou uma tendência ascendente, mesmo com as atividades de controle. Atransmissão apresenta comportamento cíclico, intercalando anos com altas e baixas incidências Os picos das epidemias coincidem com as estações chuvosas e o fator de risco mais importante tem sido morar ou circular em áreas onde estejam ocorrendo casos $^{3-6}$.

A expansão da dengue aponta para a necessidade da reestruturação da vigilância epidemiológica, mudança das políticas de controle, inclusão das realidades municipais, gestão ambiental e integração de outros setores da sociedade ${ }^{5,7}$.

Os índices de infestação predial (IIP) e pluviométrico têm sido úteis como indicadores na transmissão da dengue, na infestação de adultos, bem como na intervenção com medidas de controle ${ }^{8}$. Quando os valores de IIP estão próximos de 1\%, indicam baixo risco de transmissão. Contudo, há de se considerar múltiplos fatores condicionantes e determinantes como temperatura, sazonalidade, comportamento do vetor, aspectos sócios culturais e imunológicos da população ${ }^{9-11}$.

Este trabalho teve como objetivo evidenciar a existência de associação entre o índice predial, a pluviosidade e os casos de dengue.

\section{MÉTODOS}

OEstado de Goiás é constituído de 246 municípios, nos quais as atividades de controle são realizadas de forma descentralizada, em ações do Sistema Único de Saúde (SUS). 
A investigação de larvas de Aedes aegypti foi realizada em todos os municípios goianos, em 1.165.639 imóveis, de acordo com a metodologia preconizada pelo Ministério da Saúde 7 . As larvas encontradas foram acondicionadas em álcool a $70 \%$, e enviadas ao laboratório de entomologia do município para a identificação. Onde não havia essa estrutura foram encaminhadas ao município mais próximo.

O índice de infestação predial foi obtido através da divisão do $\mathrm{n}^{\circ}$ de imóveis com Aedes aegypti pelo $\mathrm{n}^{\circ}$ de imóveis inspecionados $\mathrm{x}$ 100, e os dados foram tabulados em planilha do Programa de Febre Amarela e Dengue (PCFAD), em cada município, e repassados à Secretaria de Estado da Saúde de Goiás.

A notificação dos casos suspeitos foi feita através da Ficha de Investigação Epidemiológica, ao Sistema Nacional de Agravos de Notificação (SINAN). Os municípios que registravam os primeiros casos enviavam material para a regional de saúde e, desta, ao Laboratório Central (LACEN/GO), para exame sorológico confirmatório pela técnica MAC-ELISA para captura de anticorpos IgM antidengue.
Os dados pluviométricos foram fornecidos pelo Instituto Nacional de Meteorologia (INMET), Regional de Goiás, em tabelas, e depois transformados em gráficos através do Programa Excel. As variáveis contínuas foram analisadas através do teste $t$.

Para analisar a associação entre o índice larval, a pluviosidade e a incidência de casos utilizou-se o coeficiente de correlação de Pearson.

\section{RESULTADOS}

O índice de infestação predial de Aedes aegypti mostrou uma grande variação entre os meses, no período de 2001 a 2005, conforme os períodos sazonais de seca e de chuva. Constataram-se associação positiva entre o IIP, a pluviosidade média e a incidência de casos de dengue, com $\mathrm{p}<0,05$. Uma forte associação se expressou em onda (Figura 1), com seus picos em épocas de maiores IIP, pluviosidade e incidência de dengue. Contudo, alguns municípios foram indenes à infestação de Aedes aegypti e à transmissão de dengue (Figura 2).

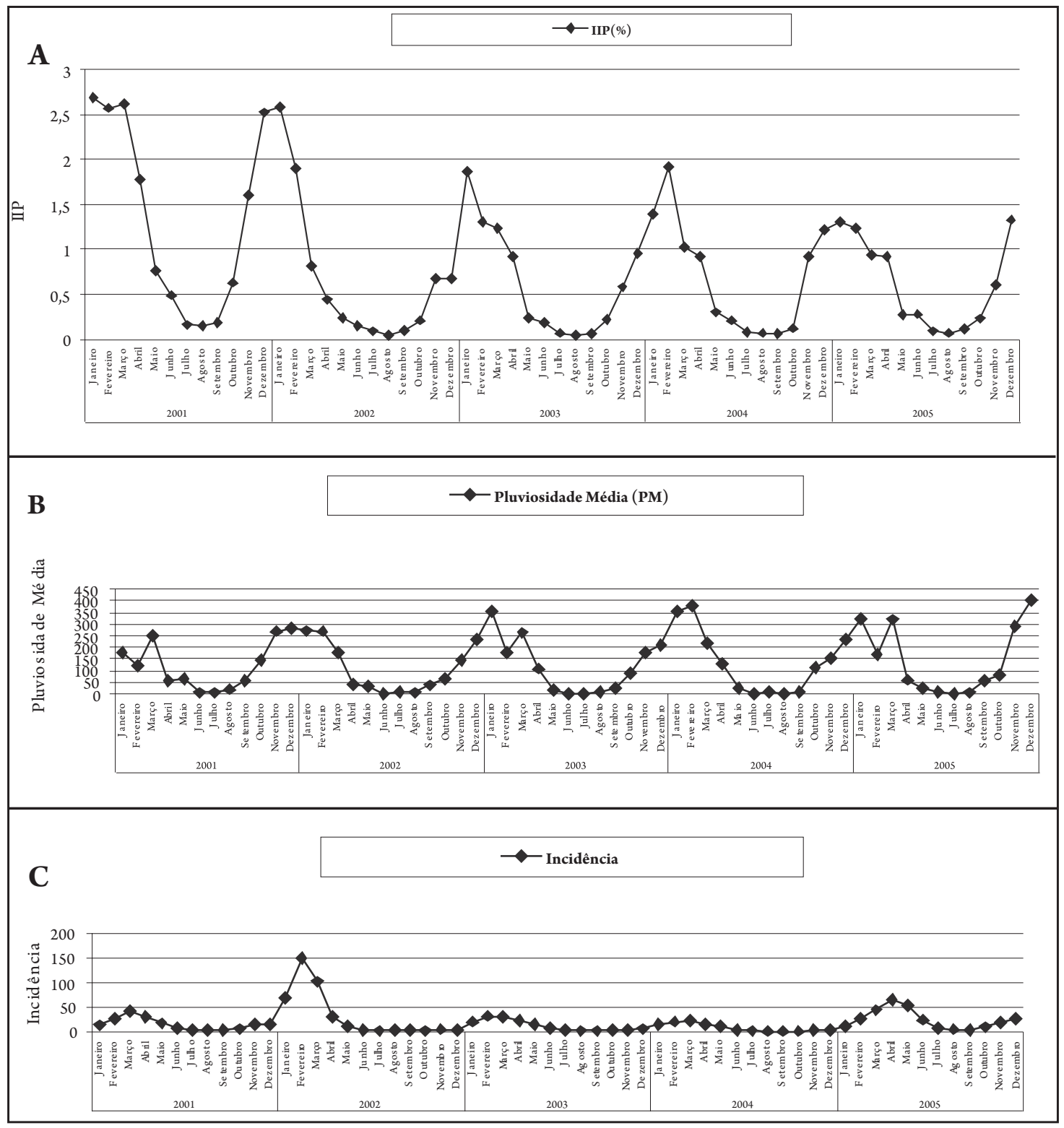

FIGURA 1 - A: Índice de infestação predial de Aedes aegypti (IIP). B: pluviosidade média (PM). C: incidência de casos de dengue (ICD), no Estado de Goiás. 


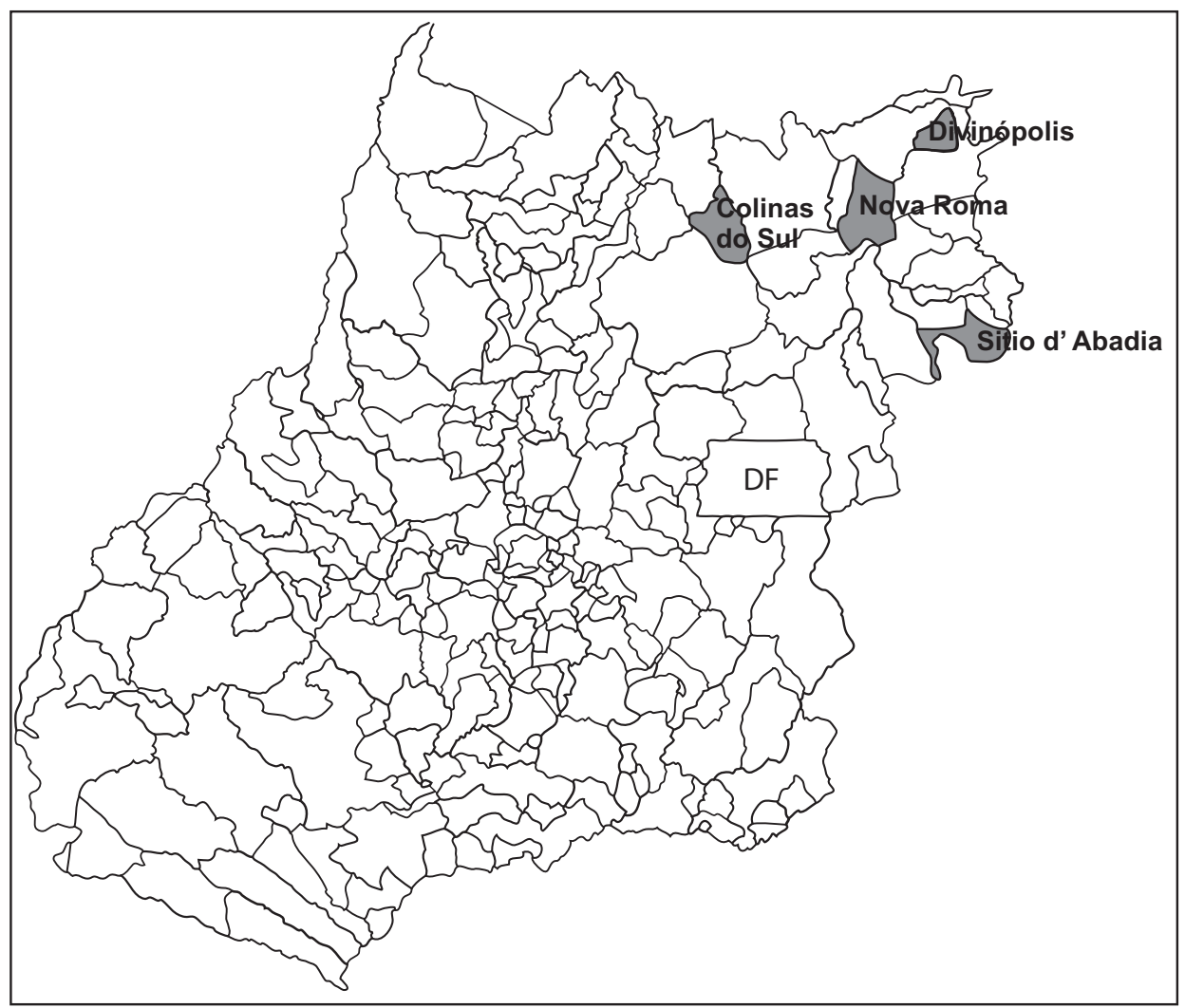

FIGURA 2 - Municípios do Estado de Goiás, indenes de Aedes aegypti, até o ano de 2005.

Em 2005, a infestação pelo Aedes aegypti atingiu 97,9\% dos municípios goianos. Apesar disso, a transmissão de dengue ocorreu, aproximadamente, na metade deles (Tabela 1). Os casos de dengue foram contínuos e crescentes, de janeiro a março de cada ano, declinando de abril a maio, quando ocorreu a interrupção na maioria dos municípios. O número de casos voltou a subir a partir de outubro, com exceção do ano de 2002. Nos meses de novembro e dezembro de todos os anos estudados, os casos se mantiveram em elevação. No período da seca, houve grande redução do IIP de Aedes aegypti, contudo, a transmissão de dengue foi contínua apenas na região metropolitana de Goiânia. A partir de 2004, a transmissão tornou-se contínua também em Minaçu, e no ano seguinte, em Rio Verde e Uruaçu, com expressiva epidemia no período de janeiro a março do ano seguinte.

TABELA 1 - Índice de infestação predial de Aedes aegypti, número de casos de dengue e incidência anual por 100.000 habitantes, de acordo com a pluviosidade dos períodos, entre 2001 e 2005.

\begin{tabular}{lrrrrr}
\hline & $\mathbf{2 0 0 1}$ & $\mathbf{2 0 0 2}$ & $\mathbf{2 0 0 3}$ & $\mathbf{2 0 0 4}$ & $\mathbf{2 0 0 5}$ \\
\hline Precipitação (mm)-chuvoso & 193 & 188,5 & 215,4 & 244,9 & 260,9 \\
Precipitação (mm) - seco & 55,3 & 27,9 & 27 & 32,5 & 29,3 \\
IIP: período chuvoso & 1,95 & 1,14 & 1,14 & 1,23 & 1,06 \\
IIP: período seco & 0,37 & 0,12 & 0,13 & 0,14 & 0,18 \\
ICD: período chuvoso & 144,49 & 367,86 & 115,85 & 80,48 & 192,5 \\
ICD: período seco & 41,75 & 27,25 & 31,47 & 20,13 & 105,1 \\
NCD: período chuvoso & 7.393 & 19.167 & 6.148 & 4.348 & 10.821 \\
NCD: período seco & 2.136 & 1.420 & 1.670 & 1.088 & 5.904 \\
Municípios com transmissão & 136 & 133 & 89 & 86 & 101 \\
\hline
\end{tabular}

IPP: índice de infestação predial, ICD: incidência anual por 100.000 habitantes, NCD: número de casos de dengue.
No período chuvoso, a incidência de casos de dengue foi de 115,8 por 100.000 habitantes, sendo $22 \%$ desse total só no mês de fevereiro. A elevada percentagem do número de casos, nos quatro primeiros meses, mostrou a influência desse período pela elevação do IIP do Aedes aegypti (Tabela 1 e Figura 1). A região metropolitana de Goiânia foi responsável por 80,6\% dos casos de dengue.

\section{DISCUSSÃO}

Estudos da associação dos fatores pluviosidade, índice de infestação predial e o número dos casos de dengue são raros. No entanto, já foi demonstrada maior incidência de dengue na estação chuvosa e nas altas temperaturas, quando aumentam a longevidade do Aedes aegypti e a possibilidade de transmissão ${ }^{3,12-16}$. Foi demonstrada também a associação entre dengue, estações chuvosas, altas temperaturas, altitudes e ventos ${ }^{17-19}$. No Estado de São Paulo, a temperatura atuou como fator modelador do processo de infestação por Aedes aegypti, porém os índices pluviométricos tiveram pequena influência ${ }^{20}$.

A associação significativa entre incidência de dengue e o IIP, deste trabalho, também foi notada em Belo Horizonte ${ }^{9}$. No período estudado de 2001 a 2005, a incidência de dengue em Goiás acompanhou os períodos de chuva e seca, com maior quantidade de casos nos meses de janeiro a abril, exceto em 2005, quando se estenderam até o mês de maio. Nesses meses, o IIP e a incidência de casos de dengue foram mais elevados, tanto por município, quanto no estado inteiro, confirmando a hipótese de que a transmissão ocorre quando a densidade do vetor está elevada. Há estudos que referem os IIP por semestre ${ }^{9,21}$, nesse procedimento misturam parte da estação chuvosa com a seca, sendo o índice maior no primeiro do que no 
segundo, e não nos períodos de maiores ou menores pluviosidade. Nesses estudos, os maiores valores do IIP foram entre 1,33 e 2,76\% e os menores abaixo de $0,45 \%$, sendo seus valores maiores do que os desse trabalho.

Até o momento, em Goiás, estão circulando simultaneamente três sorotipos do vírus (Den-1,2 e 3). Estudos mostram que há circulação viral com IIP em torno de 1\%, ou acima desse índice ${ }^{9,13}$. Em Goiás, a transmissão de dengue ocorria com o IIP>1\%. Em 2001, dos 136 municípios com transmissão, 58 tiveram IIP $<1 \%$. Mas, o maior número de casos de dengue foi no período chuvoso, com $77,5 \%$ dos casos, e o restante no período seco $(22,5 \%)$. No período chuvoso de 2002, ocorreu uma grande epidemia de dengue, a maior registrada até o momento, com 20.587 casos, e o IIP observado foi de 1,14\%. A região metropolitana de Goiânia foi responsável por mais de $80 \%$ do total de casos. Provavelmente, o aumento tenha sido maximizado pela introdução do sorotipo Den-3, acrescido aos dois já existentes em circulação, visto que diminuiu o número de municípios com transmissão. Outro dado que corrobora esse raciocínio foi o fato da maior prevalência de Den-3 na região metropolitana de Goiânia que representa a maior concentração populacional do estado de Goiás. Nessa região, e no município de Minaçu, a transmissão de dengue foi contínua a partir de 2003.

Parece lícito supor que isso tenha sido um fator determinante na elevação do número de casos de dengue, devido ao fluxo de dispersão para outros municípios, principalmente para os próximos, que fazem fronteiras. A dispersão passiva pode ocorrer em função dos inter-relacionamentos comerciais, ou pela procura de empregos, assistência à saúde e educação. Esse fluxo de pessoas contribuiu para a dispersão dos sorotipos do vírus de dengue da capital para as cidades contíguas (Aparecida de Goiânia) ou próximas (Trindade e Senador Canedo), elevando o número de casos de dengue.

Outra epidemia importante de dengue em Goiás aconteceu em 2005, com 16.725 casos, em 101 municípios. Isso significou um aumento de casos de 307,6\% em relação ao ano anterior, e de $11 \%$ do número de municípios. A transmissão foi contínua em Goiânia, Aparecida de Goiânia, Trindade, Senador Canedo, Rio Verde e Uruaçu. Novamente se repete o fato de que a continuidade da transmissão faz aparecer acentuada elevação do número de casos em outubro.

A associação positiva entre o IIP e a pluviosidade, e o IIP e a incidência de casos, sugere que o IIP seja um fator crítico significativo na transmissão de dengue, mesmo sabendo que a pluviosidade não é fator determinante ao desaparecimento do mosquito ${ }^{20}$ porque não depende exclusivamente dos criadouros que surgem com o período chuvoso. O Aedes aegypti mantém o ciclo de vida no período seco, através de criadouros artificiais permanentes, como caixas d'água e caixas de passagem do sistema hidrossanitário. O mosquito sobrevive em densidade baixa, mas o suficiente para manter a transmissão contínua, embora em declínio, confirmada pelos municípios com casos de dengue no período seco.

\section{AGRADECIMENTOS}

Aos chefes e equipes dos Núcleos de Apoio ao Controle de Endemias da Secretaria de Estado da Saúde de Goiás pelo imprescindível apoio nos trabalhos de campo. À Dra Elizabeth Carmen Duarte pelas contribuições nas análises estatísticas.

\section{CONFLITO DE INTERESSE}

Os autores declaram não haver nenhum tipo de conflito de interesse no desenvolvimento do estudo.

\section{REFERÊNCIAS}

1. World Health Organization. Dengue/dengue haemorrhagic fever prevention and control. Regional Office for South-East Asian 2003; 1-33.

2. Silva IG, Araújo ESO, Silva HHG, Soares AW, Cantuária PB. Ocorrência de Aedes aegypti (Linnaeus, 1762) (Diptera, Culicidae) em Goiânia. An Soc Entomol Brasil $1991 ; 20: 459-460$.

3. Câmara FP, Gomes AF, Santos GT, Câmara DCP. Clima e epidemias de dengue no Estado do Rio de Janeiro. Rev Soc Bras Med Trop 2009; 42:137-140.

4. Gubler DJ. Dengue and dengue hemorrhagic fever. Clin Microbiol Rev 1998; 11:480-496.

5. Organización Panamericana de la Salud. Dengue y dengue hemorrágico em las Américas, guias para su prevención y control. Publicacion Científica no 548, 2002.

6. Gomes AC. Medidas dos níveis de infestação urbana para Aedes (Stegomyia) aegypti e Aedes (Stegomyia) albopictus em programa de vigilância entomológica. IESUS 1998; 7:49-57.

7. Koopman JS, Prevots DR, Marin MAV, Gomes-Dantes H. Determinants and predictors of dengue infection in Mexico. Am J Epidemiol 1991; 133:11681178 .

8. Glasser CM, Gomes AC. Infestação do Estado de São Paulo por Aedes aegypti e por Aedes albopictus. Rev Saúde Pública 2001; 34:570-577.

9. Corrêa PRL, França E, Bogutchi TF. Infestação pelo Aedes aegypti e ocorrência da dengue em Belo Horizonte, Minas Gerais. Rev Saúde Pública 2005; 39:33-40.

10. Donalísio MR, Glasser CM. Vigilância epidemiológica e controle de vetores do dengue. Rev bras epidemiol 2002; 5:259-272.

11. Tauil PL. Aspectos críticos do controle do dengue no Brasil. Cad Saúde Pública 2002; 18:867-871.

12. Fundação Nacional de Saúde. Plano Diretor de Erradicação do Aedes aegypti no Brasil, Brasília; 1996.

13. Fundação Nacional de Saúde. Programa Nacional de Controle da Dengue-PNCD, Brasília; 2002.

14. Goh KT. Changing epidemiology of dengue in Singapore. Lancet 1995 346:1098.

15. Gould DJ, Mount GA, Scanlon JE, Ford HR. Ecological control of dengue vectors on an island in the Gulf of Thailand. J Med Entomol 1970; 4:499-508.

16. Siqueira Jr JB, Martelli CMT, Coelho GE, Simplicio ACR, Hatch DL. Dengue and Dengue Hemorrhagic Fever, Brasil, 1981-2002. Emerg Infect Dis 2005; $11: 48-53$

17. Foo LC, Lee HL, Fang R. Rainfall, abundance of Aedes aegypti and dengue infection in Selangor, Malaysia. Southeast Asian J Trop Med Public Health 1985; $16: 560-568$.

18. Ribeiro AF, Marques GRAM, Voltolini JC, Condino MLF. Associação entre incidência de dengue e variáveis climáticas. Rev Saúde Pública 2006; 40:671 676.

19. Watts DM, Burke DS, Harrison BA, Whitmire RE. Effect of temperature on the vector efficiency of Aedes aegypti for dengue 2 virus. Am J Trop Med Hyg 1987; 36:143-452.

20. Fundação Nacional da Saúde. Boletim Epidemiológico. Ministério da Saúde. Brasília; 1999.

21. Teixeira MGL, Barreto ML, Costa MCN, Ferreira LDA, Vasconcelos PFC. Avaliação de impacto de ações de combate ao Aedes aegypti na cidade de Salvador, Bahia. Rev Bras Epidemiol 2002; 5:108-115. 